\title{
Efficiency increasing by development of district heating systems operational modes using graphical analysis
}

\author{
Vyacheslav V. Tokarev ${ }^{1, *}$, Aleksandr V. Alekseev ${ }^{1}$ and Zoya I. Shalaginova ${ }^{1}$ \\ ${ }^{1}$ Melentiev Energy Systems Institute of Siberian Branch of the Russian Academy of Sciences (ESI SB \\ RAS), 130 Lermontov Str., Irkutsk 664033, Russia
}

\begin{abstract}
The tasks of analyzing, developing and justifying of the district heating systems (DHS) operation modes arise at different stages of their life cycle. The article provides a brief description of the tasks, steps and methods for calculating and analyzing DHS modes, which are implementing as subtasks of the "ANGARA-HN" software. Special attention is paying to the automation of analysis processes. The developed computational and graphical software tools for parameters analyzing of the DHS operating modes consists of the means: analyzing of the initial information, calculating the values of the mode parameters and analyzing results of the calculation. Various tools allow you to visualize the distribution of selected parameters values on the graphical city plan, highlight bottlenecks, building pressure distribution graphs. The developed software designed to automate the workplaces of regimes group engineers at operational enterprises or for designer workers, when developing and justifying development schemes for urban DHS. Tools for analysis and graphical visualization significantly increase the efficiency of work by reducing the time of analysis, development and justification of operating conditions, as well as improve the quality of the results.
\end{abstract}

\section{Introduction}

Modern DHS of large cities and settlements play an important socio-economic role in providing thermal energy and hot water to the civil customers, industry, public and social facilities. The effectiveness, reliability and quality of their operation is directly dependent on the competent organization of the operation modes of DHS. The tasks of modes developing are characterized by significant complexity due to their large dimension, uncertainty and inconsistency of information about their actual state, changes of the thermal energy demand and network structure over time. In modern conditions, the effective solution of these problems becomes impossible without the use of computer technology and advanced specialized information technologies.

\footnotetext{
* Corresponding author: tslava@isem.irk.ru
} 
The tasks of analyzing, developing and justifying the modes of operation of the DHS arise at different stages of their life cycle. During designing and making of development plans for the DHS [1-3], calculations are performed in order to: 1) analyze the capacity of the existing system for prospective loads; 2) verification of the admissibility of the work of the center in non-design modes; 3 ) analysis and justification of the reliability of the supply of consumers after the implementation of the design options of DHS. For planning modes for the upcoming heating season, the development and justification of adjustment activities $[4,5]$ during the operation of DHS calculations are carried out in order to: 1) analyze the allowability and rationality of existing conditions; 2) identifying the causes of existing violations, "system bottlenecks"; 3) development rational zoning of DHS schemes; 4) the development of schedules for pumping stations, tanks and other constructures; 5) determine the rational places of installation of regulators, determine the values of the parameters supported by them; 6) determine the network capacity to connect new consumers; 7) analysis of the consequences of possible accidents and the development of dispatching schedules for their elimination; 8) analysis of network bandwidth reserves, etc. In dispatching control [6-9], mode calculations are used to substantiate and verify management decisions. For example, it can used in the period of elimination of consequences of accidents or working for repair and restoration.

In this article, against the background of a brief description of the tasks, stages and methods for calculating and analyzing DHS modes, the results of the development of information and computing technologies for computer modeling of the DHS developed in ESI SB RAS based on the "Angara" information-computing environment (ICE) are described. At the same time, special attention is paying to automating the analysis processes: information, calculation results, and the actual operation modes of DHS. These issues are not sufficiently developed in the existing applied software and computing complexes, but they are crucial for the efficiency, reliability and operatively of the analysis and decision-making processes when organizing modes.

\section{Methodical background}

The development of mathematical models and methods for calculating stationary and dynamic, hydraulic and thermo-hydraulic DHS modes has a long history [10-12]. Depending on the type of systems of equations describing the mode (algebraic, implicit, differential, etc.) are distinguishing models with lumped, variable (adjustable) and distributed parameters [1]. In modeling, quite often various methods of decomposition are used $[4,13]$, as well as simplifications and reductions in the dimension of the models involved at the level of simplification of computational schemes. Such methods are focused on special properties: design tasks, reconstruction $[14,15]$, operational control $[6,16]$; is attracted models of stationary $[6,14-16]$ or dynamic $[8,9,17,18]$ modes; modeling purposes. The basic methods of analysis are the general methods for calculating the steady flow distribution: pressure method [1,19,20], flows in cycles method [1,20], global gradient method [21], and others. Recent work in this direction is devoted to modeling and organizing complex multi-loop pipeline systems [15,22-24].

The authors of the article within the framework of the scientific direction developed in ESI SB RAS over the several decades - Theory of Hydraulic Circuits [1,2] have accumulated a unique experience in creating methodological and software solutions for calculating and optimizing pipeline systems of various types and purposes [25-27], which is implemented in dozens of research, design and operational enterprises in Russia, as well as abroad. 


\section{Stages and features of the development of DHS modes}

The process of mode developing divided into the following stages: 1) initial information analysis; 2) existing mode and network bandwidth analysis; 3) analysis of violations in the mode; 4) measures development for the organization of an allowable settlement mode (entry of a mode into an acceptable area). On each stage is analyzing its own set of information.

The processes of analysis, development and justification of the regimes require a large amount of work on the preparation and refinement of the initial information about the DHS schemes and parameters and conducting multivariate calculations. No less difficult is the stage of analysis of the obtained calculation results, especially for large DHS, when the number of mode parameters for the network elements amounts to many hundreds of thousands, and each of them should be checking for allowable by several indicators.

The analysis of the current regime, as well as the development of controls, is based on calculations of hydraulic and temperature regimes. Usually such calculations are dividing into adjustment and test calculations. The main purpose of the adjustment calculation is to find such control parameters that will ensure the passage of specified water flow rates through DHS subscriber systems while respecting the technological limitations on the heat carrier parameters - pressures in all system nodes, pressures on consumers' inputs and heat carrier velocities.

For analyzing the state of heating networks, the temperature mode is important to take into account: cooling of the heat carrier along the transportation route; deficiency of heat energy at consumers; selection of measures to heat losses compensating in the network (by increasing flow rate of the heat carrier or by changing of temperature graph). The result of the adjustment of the heat network is the mode in which the specified heat loads of all consumers and other restrictions arising from the requirements of acceptable operating conditions of the equipment are ensured.

Entering a mode into an acceptable area is a difficult task and requires the use of multivariate or optimization calculations according to technological and economic criteria. In practice, it is not always possible to create an acceptable and, moreover, optimal mode only at the expense of regulation means. In some cases, it may be necessary to reconstruct the system as a whole or its individual elements.

Test calculations of operating modes are performing in order to determine deviations of the mode parameters, including the degree of consumers' supply, from the required values in non-design or non-standard conditions. Non-standard conditions include, for example, emergency modes.

\section{Methods for analyzing DHS modes and data when calculating operational modes}

There is a huge number of methods for analyzing and processing data. For the analysis of the operating mode statistical methods of data processing (searching for extreme values of parameters and their plausibility expert analysis), visualization (highlighting color and size in the diagram) and checking the restrictions on parameters are most importance.

Statistical processing methods are useful for finding outliers of parameter values from the average level, which, as a rule, indicates an error in the data.

By help of size visualization (width) of the pipeline section, one can analyze the distribution: the diameters of the pipeline sections, the service life of the pipeline, the roughness of the pipelines, the value or proportion of local losses in the pipelines, etc. The size of the element in the diagram can be proportional to the heat load, geodetic mark, etc. With the help of the color of the pipeline section, it is possible to analyze the zone of 
influence of the heat source, the distribution of types of laying of pipelines, etc. The color of the node of the thermal network can be estimate the distribution of categorizing consumer, temperature level at the consumer, the level of the static pressure and others.

In accounting practice, validation of values is usually performing by means of spreadsheets. However, in our case, it is possible to determine the location of elements with critical values of parameters on the network plan, focused on the terrain plan. Thus, the visualization of the control values indicates the location of the node or pipeline element with violation of mode restrictions from the allowability requirements.

\section{Universal technology for analysis of DHS modes}

Since 2000, the software of Information and Computing Environment (ICE) "Angara" has been developing at ESI SB RAS for modeling pipeline systems of various types and purposes. ICE has a complex of graphic, informational and analytical functions necessary for creating, editing and analyzing networks of various types of systems [28]. On its base from 2003 to 2018 several modifications of the "ANGARA-HN" have been released, which is an integration of the ICE and software-computer complex (SCC) for calculating the thermal-hydraulic modes of DHS $[29,30]$.

The ICE is open for functions (through the COM interface) and data. It has configured to work with various DBMS. For example, MS Access is using for local workplaces, for network multi-user operation are using MS SQL SERVER. This openness allows users independently develop connection modules without participation of ICE developers.

Three tools for data analysis have been implementing at the ICE "Angara".

1) "Numerical analysis" is intending for processing digital data and allows you to find extreme, average values, as well as their sum by the selected element parameter. This feature is useful for finding outliers that are often associated with data errors. Such an analysis can be applied both to the scheme as a whole and to an arbitrary selected fragment of the network. In addition to the calculation, it is possible to set the same values in the specified database fields for selected elements of a certain type.

2) "Graphical analysis" - highlighting the elements of a pipeline network in the scheme by animation, color and/or size. You may apply at the same time a selection for any element both by size and by color. The function interface has three tabs for highlighting by color and size for all and part of elements for which the specified condition is realizing.

3) Automation of using of graphical analysis functions. In the ICE, a library of "templates" for the use of graphic functions has been developed. It allows, once having created a selection template on the scheme of certain parameters or their group, to save it in the database for subsequent repeated use on arbitrary design schemes. Editing templates allows you to make changes to them depending on the needs of the calculations and save these changes in the database. Thus, typical operations on the graphical interpretation of the design schemes data are performed by "pressing of one button".

\section{Applied use of graphical analysis tools of the ICE "Angara" for increasing the efficiency of developing DHS modes}

Analysis of the initial information. It is possible to select the elements of the scheme by color and size using any data values, however, the experience of using ICE revealed the most informative cases of visualization by size (width) of pipeline sections. This is an analysis of the distribution: diameters of pipeline sections, pipeline service lifetime, pipeline roughness, the value or proportion of local losses in pipelines, etc. The size of the element on the diagram should be analyzed: load value, geodetic elevation, etc. Using the 
color of the pipeline section for visualization of zone of heat source influence, distribution of types of laying and pipeline materials, pipeline affiliation to different enterprises, etc. The color of the node network element - the category of consumers, the level of pressure or temperature at consumers, and others.

Analysis of restrictions violations on the parameters of the mode. The parameters of the DHS mode, in accordance with regulatory documents, are limiting by the strength conditions of the equipment, non-boiling conditions of the heat carrier, prevention of vibration, air isolation of pipelines, cavitation of pumps, etc. The checking of constraints [5, 31] on pressures and temperatures in network nodes and on DHS facilities (heat sources, pump stations, central thermal stations and consumer thermal stations), speeds on network pipeline sections, etc. was implemented at the "Angara-HN". When a multi-level structure of the DHS model constraints are checking for each hydraulically unconnected fragment of one level. When calculating distribution networks in the "generalized source" or central thermal station element, constraints are finding that are used to analyze the admissibility on the main network level.

Graphic interpretation of the calculation results. As a result of the calculation of the DHS mode [5,13,32], the flow rates distribution, pressures and temperatures is calculated for all network elements, as well as the parameters of regulators and other throttling devices. The piezometric graph (PG) is a graphic representation of the change in the full heads in the pipelines along a given route. The built-in module of such a graph in the "Angara-HS" software allows you to display an extended list of specifications: geodetic elevations of land, building heights, measurement results, gauge pressures and static pressures at network nodes, flow rates, speeds, specific losses in network sections, etc. With a multi-level organization of the computer model, it is possible to build a piezometer from any node of main network to a consumer connected to the distribution network. The length of the route can be significant. To increase visibility, the possibility of PG scaling is providing in proportion to the length of the route. In this case, areas with large specific pressure losses are visible. Displaying a graph not to scale allows you to see all the specifications of PG. PG provides an opportunity not only to determine the locations of possible violations of permissible pressures or delivered pressure head, but also to detect their causes, for example, bottlenecks in the network for which a sharp pressure drop is observed. PG allows detecting nodes with violations of pressure limitations, pipelines with violation of speed limits, or pipelines with high specific pressure losses. Also improves the visibility of the calculation results and the speed of analysis by visualization of information on a graphical network scheme. By size (width) of pipeline sections clearly shows the distribution: delivered pressure heads in pipeline nodes, heat carrier flow rates, specific pressure losses in pipelines, etc. The size of the element in the scheme should analyze the distribution of delivered pressure heads at consumer inputs, of gauge heads at supply and return nodes, etc. The color of the pipeline section is appropriate for visualize the speed of the heat carrier in the pipeline, the zone of influence of the heat source, etc. The color of node like elements is useful for showing the distribution of air temperatures inside of consumers, heat carrier temperatures in nodes of the network, etc.

\section{Conclusion}

The investigation of identify processes that require automation when the development of DHS operating modes, allowed to implement the appropriate tools in the ICE "Angara" software. Software and computing tools for analyzing the parameters of DHS operational modes showed its effectiveness in analyzing data using: visualizing the distribution of values of selected parameters on a graphical scheme of the city, highlighting bottlenecks, plotting PG and characteristics of engineering systems objects. Developed "Angara-HN" 
software to automate the calculation and analysis of DHS modes. Implemented in them analysis and graphical visualization tools increase the efficiency of development by accelerating the working process and improving the quality of the resulting modes. Recommendations were developed on the automation of graphical analysis by means of ICE, both of the initial data and the results of the calculation of large DHS hierarchical structures with intermediate levels of control.

The research was carried out within the project III.17.4.3 of the Fundamental research program of SB RAS (AAAA-A17-117030310437-4) with finance support of RFBR and the Government of Irkutsk Region in the framework of research project № 17-48-380021

\section{References}

1. A.P. Merenkov, V.Ya. Khasilev, Theory of hydraulic circuits (Moscow:Nauka,1985)

2. A.P. Merenkov, E.V. Sennova, et al. Mathematical modeling and optimization of heat, water, oil and gas supply systems (Novosibirsk:Nauka, 1992)

3. V.A. Stennikov, E.A. Barakhtenko, D.V. Sokolov, Energy Systems Research, 1, 1, 84 (2018)

4. V.V. Tokarev, Z.I. Shalaginova, Thermal Engineering, 63, 1, 68 (2016)

5. N.N. Novitsky, Z.I. Shalaginova, et al., Izvestiya RAS: Energetika. 1, 12 (2018).

6. N.N. Novitskii, Thermal Engineering, 56, 12, 1024 (2009).

7. E. Guelpa, C. Toro, et al., Energy, 102, 586 (2016)

8. M. Vesterlund, A. Toffolo, J. Dahl, Energy, 126, 53 (2017)

9. E. Guelpa, A. Sciacovelli, V. Verda, Energy, DOI: 10.1016/j.energy.2017.07.177 (2017)

10. M.M. Andriyashev, Engineering calculation of the water supply network (1932)

11. V.G. Lobachev, Sanitary equipment, 2, 8 (1934)

12. H. Cross, Eng. Exp. Station of Univ. of Illinois, 286, 29 (1936)

13. Novitsky N.N., et al., Energy, DOI: 10.1016/j.energy.2018.02.070 (2018)

14. A. Lazzaretto, A. Toffolo, et al., Energy, 35, 2, 1157 (2010)

15. M. Vesterlund, J. Dahl, Energy Conversion and Management, 89, 555 (2015)

16. A. Di Nardo, A. Cavallo, et al., Procedia Engineering, 154, 1275 (2016)

17. G. Schweiger, P.-O. Larsson, et al., Energy, 137, 566 (2017)

18. B.S. Gilani, M. Bachmann, M. Kriegel, Energy Procedia, 122, 385 (2017)

19. N.N. Novitsky, Izvestiya RAS: Energetika, 6, 56 (2013)

20. N.N. Novitsky, V.V. Tokarev, Izvestiya RAS: Energetika, 2, 88 (2001)

21. E. Todini, S. Pilati, Computer Applications in Water Supply, 1, 1 (1988)

22. Sh. Zhou, H. Li, et al., Applied Thermal Engineering, 119, 215 (2017)

23. Y. Wang, Sh. You, et al., Energy, 126, 603 (2017)

24. V.V. Tokarev, Thermal Engineering, 65, 6, 400 (2018)

25. N.N. Novitsky, et al., Pipeline systems in energy sector (Novosibirsk: Nauka, 2014)

26. N.N. Novitsky, et al., Pipeline systems in energy sector (Novosibirsk: Nauka, 2015)

27. N.N. Novitsky, et al., Pipeline systems in energy sector (Novosibirsk: Nauka, 2017)

28. A.V. Alekseev, N.N. Novitsky, Scientific Bulletin of NSTU, 3, 26 (2017)

29. N.N. Novitsky, Z.I. Shalaginova, et al., Izvestiya RAS: Energetika. 1, 12 (2018)

30. N.N. Novitsky et al., Proc. of Univ. Investment. Construction. Real estate, 8, 4, 139 (2018)

31. N.N. Novitsky et al., Proceedings of IrSTU, 11, 126 (2018).

32. V.V. Tokarev, Z.I. Shalaginova, Proceedings of IrSTU, 12, 240 (2011) 